\title{
New Hydrophilic, Composite Membranes for Air Removal from Water Coolant Systems
}

\author{
${ }^{1}$ Stephen M.C. Ritchie*, ${ }^{1}$ Qiang Luo, ${ }^{1}$ Salina S. Curtis, \\ ${ }^{2}$ Jon B. Holladay, and ${ }^{2}$ Dallas W. Clark \\ ${ }^{1}$ Department of Chemical Engineering, University of Alabama \\ ${ }^{2}$ Flight Projects Directorate, FD24, Marshall Space Flight Center
}

\section{ABSTRACT}

Liquid coolants are commonly used as thermal transport media to increase efficiency and flexibility in aerospace vehicle design. The introduction of gas bubbles into the coolant can have negative consequences, including: loss of centrifugal pump prime, irregular sensor readings, and blockage of coolant flow to remote systems. One solution to mitigate these problems is the development of a passive gas removal device, or gas trap, installed in the flight cooling system. In this study, a new hydrophilic, composite membrane has been developed for passage of the coolant fluid and retention of gas bubbles. The trapped bubbles are subsequently vented from the system by a thin, hydrophobic, microporous membrane. The original design for this work employed a homogeneous membrane that was susceptible to fouling and pore plugging. Spare gas traps of this variety have degraded during storage, and recreation of the membranes has been complicated due to problems with polymer duplication and property variations in the final membranes. In this work, replacements have been developed based on deposition of a hydrophilic polymer on the bore-side of a porous polyethylene (PE) tube. The tube provides excellent chemical and mechanical stability, and the hydrophilic layer provides retention of gas bubbles. Preliminary results have shown that intimate contact is required between the deposited layer and the substrate to overcome material differences. This has been accomplished by presoaking the membrane tube in the solvent to raise its surface energy. Polymer solutions of various concentrations have been used to promote penetration of the polymer layer into the porous substrate and to control separation layer thickness. The resulting composite membranes have shown repeatable decrease in nitrogen permeability, which is indicative of a decrease in membrane pore size. Studies with water permeation have yielded similar results. We have observed some swelling of the added polymer layer, which causes a slight decrease in membrane pore size, and should result in improved bubble retention. Preliminary studies have also been performed on gas retention in flowing systems. Initial results have been promising, with negligible gas permeation for the coated membranes compared to $100 \%$ gas permeation in the uncoated tube.

\section{INTRODUCTION}

The introduction of gas bubbles is inevitable in fluid coolant loops containing orbital replacement units (ORUs). Non-evacuated gases in the ORUs and dead-spaces in quick disconnects (QDs) are the primary sources of gas bubbles. These must be removed from the coolant loop, as their presence can have several negative effects, including: loss of centrifugal pump prime, interference with sensor readings, and 
blockage of fluid flow to remote systems. The removal of gas bubbles in microgravity is non-trivial because of the lack of buoyancy. Therefore, creatively designed gas traps must be used to remove these non-condensable gases from the system.

One current design involves the use of a dual-membrane system (SAE \#932162). In this case, one membrane is used to retain gas bubbles, while the other membrane is used to vent the bubbles from the system. This gas trap is currently being used on the International Space Station (ISS). It is important to note that the need for gas traps is not exclusive to the ISS; rather, they are needed in the logistics module and in future NASA projects such as the Strategic Launch Initiative (SLI) and the Orbital Space Plane (OSP). Although the original gas trap design was effective in ground tests, it has demonstrated decreased performance and susceptibility to fouling on orbit. There is a demonstrated need to find replacements for the existing gas traps on the ISS and in other NASA applications. This work builds on a redesign of the dual-membrane gas trap (SAE \#2003-01-2569) with a focus on the hydrophilic membrane material.

The material change has been addressed through the formation of a composite membrane. The membrane is formed by deposition of a thin coating of polyethersulfone (PES) in the bore of a porous polyethylene (PE) tube. The PES provides the separation ability for selective passage of water and retention of gas bubbles, while the PE tube provides the structural stability. Both the polymer and the tube are available commercially. Methods of formation, problems encountered and resolved, and preliminary results will be provided here.

\section{BACKGROUND}

A dual-membrane gas trap is currently used on the ISS. This trap is composed of an array of 84 tube sets. Individual tube sets are composed of an outer hydrophilic membrane, and an inner hydrophobic membrane. A schematic of the tube set is shown in Figure 1. Note that coolant enters the bore of the hydrophilic membrane, passes through the wall, and then exits the gas trap. Gas bubbles retained by the hydrophilic membrane adhere to the hydrophobic membrane, where they are subsequently vented to the cabin. The hydrophilic membrane has larger pores than the hydrophobic membrane. However, since its pores are filled with water, air bubbles must overcome the membrane bubble pressure to displace this fluid before they can bypass the gas trap. As long as the bubble pressure is greater than the actual pressure drop across the gas trap, bubbles are retained.

The hydrophilic membrane is critical for the capture of gas bubbles, and the bubble pressure and overall pressure drop are direct functions of the membrane material and pore size. For a fixed mass flux and membrane pore size, a more hydrophobic material will have lower water permeability than a more hydrophilic material. For example, the Nylon-11 membranes (3.6 $\mu \mathrm{m}$ pore diameter) used in the original gas traps have a water permeability of $186 \mathrm{lb} / \mathrm{ft}^{2} \mathrm{hr}$ psi. A more hydrophilic polymer, such as PES, has approximately the same permeability $\left(167 \mathrm{lb} / \mathrm{ft}^{2} \mathrm{hr} \mathrm{psi}\right)$ at a pore diameter of only 0.22 $\mu \mathrm{m}$. Therefore, a PES membrane with the same pore size as the Nylon-11 membrane would have substantially higher water permeability. 


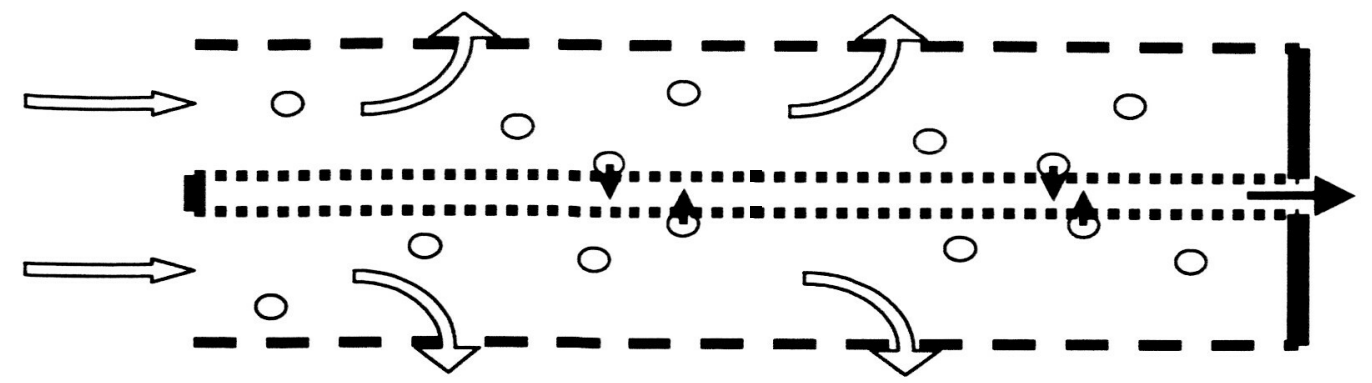

Figure 1: Schematic of membrane tube set. Outer dashed lines represent the hydrophilic membrane. Inner dashed lines represent the hydrophobic membrane.

The bubble pressure is a stronger function of pore size than membrane material. It can be calculated directly using equation 1 ,

$$
\text { Bubble Pressure }=\frac{4 k \cos \theta}{d} \sigma
$$

where $k$ is a shape factor (unitless), $\theta$ is the contact angle (degrees), $d$ is the pore diameter $(\mu \mathrm{m})$, and $\sigma$ is the liquid surface tension $(\mathrm{mN} / \mathrm{m})$. The true value of this equation is the relationship between the bubble pressure and $d$, since the other parameters are constant. Reported data modeled by this equation are shown in Figure 2. The model fits the data exceptionally well, and predicts bubble pressures of 7.3 and $51 \mathrm{psi}$, respectively, for the Nylon-11 and PES membrane discussed above. Given the similar water permeability of these membranes, a switch to PES membranes would result in a much higher tolerance for the pressure increases observed during operation of the gas traps on orbit.

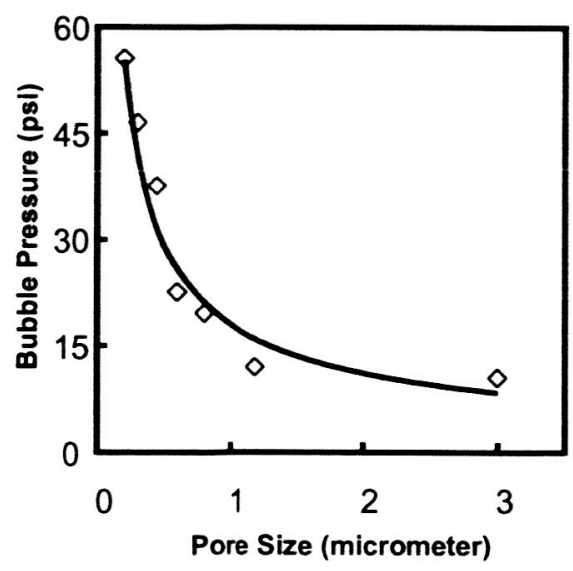

Figure 2: Bubble pressure dependence on membrane pore diameter.

In the course of this research, hydrophilic, composite membranes were formed by deposition of PES in the bore of a porous PE tube. Advancements have been made on coating procedures and methods for testing and comparison of different composite membranes. This paper will focus on lessons learned during coating experiments, 
observed decreases in membrane permeability after coating, and some preliminary work demonstrating improved bubble pressure over the substrate tube.

\section{EXPERIMENTAL}

\section{Materials}

The two samples of PE tubes used in this study were obtained from PoreX Corporation (Fairburn, GA) and Interstate Specialty Products (Leicester, MA). The PES was a commercial grade Radel A obtained from Solvay (Brussels, Belgium). Reagent grade dimethylformamide (DMF) was purchased from Fisher Scientific (Pittsburgh, PA) and used as received. Polymer solutions were made by dissolution of $10 \mathrm{~g}$ of polymer in 45 or $90 \mathrm{~mL}$ DMF, degassing, and storage in a cooler at $10^{\circ} \mathrm{C}$ until needed.

\section{Tube Preparation}

Early studies used the PoreX tube as received. The majority of coated membranes were made by first soaking the PE tube in DMF. Soak times varied between 20 minutes to 2 hours. The tubes were removed from the solvent and the exterior surface was wiped dry prior to polymer coating.

\section{Tube Coating}

Typical membrane tube coating was performed by placement of the dried (see previous) tube in a tube holder assembly. This permitted hands-free application of the polymer solution to the bore of the tube. Once placed in the holder, 1-2 pipettes of the polymer solution $(\sim 4 \mathrm{~mL})$ were applied to the bore side of the tube. Visual inspection verified uniform surface coating as evidenced by the presence of a smooth, glistening coating in the tube bore. Excess polymer was allowed to drain for various periods of time, and residual polymer at the base of the tube was wicked away with a paper towel.

\section{Phase Inversion and Membrane Drying}

The coated tubes were next placed in a beaker of still water. Immersion in water permits leaching of the solvent DMF from the coating followed by precipitation, or phase inversion, of the polymer. Distortions in the beaker liquid headspace were evidence of solvent leaching from the tube. After two minutes in the bath, the tube was rinsed directly with flowing water for 3 minutes. The tube was then allowed to soak in flowing water for 25 minutes. Flowing nitrogen through the bore dried the coated tube.

\section{Nitrogen Flux Testing}

Tubes were tested for nitrogen permeability to get a relative feel for decrease in membrane pore size after coating. Tygon $\circledast$ tubing was attached to each end of the coated tube with hose clamps. One end of this assembly was closed with a second clamp, while the other end was attached to the nitrogen supply. A pressure regulator in the line allowed for application of a constant pressure difference across the tube. The nitrogen flow rate was measured by collecting permeated gas in a water-filled graduated cylinder. As gas displaced water in the cylinder, the volume of nitrogen could be measured versus time. The flow rate was normalized by the membrane crosssectional area to calculate the nitrogen flux. Further normalization with the applied pressure results in a membrane permeability that offers a means for objective 
comparison between different coated tubes. The nitrogen flux was determined in the same way after permeation of $100 \mathrm{~mL}$ water through some of the membranes.

\section{Water Flux Testing}

Tubes were tested for water permeability to examine the effectiveness of the deposited PES film, and to examine swelling of the layer over time. Tests were performed using the set-up in Figure 3. A static pressure head was maintained in the system by changing the liquid holdup level in the feed tank. The coated membrane tubes were inserted into the system, and one end of the tube was closed off so that all water flow would be through the tube walls. Water was collected as a function of time, and the flow rate was normalized with the membrane cross-sectional area and the pressure drop to calculate the membrane permeability. Swelling tests were conducted by allowing water to flow through the membrane for a set period of time before measurement of the flow rate. Swelling of the membrane was demonstrated by a decrease in the water flow rate over time.

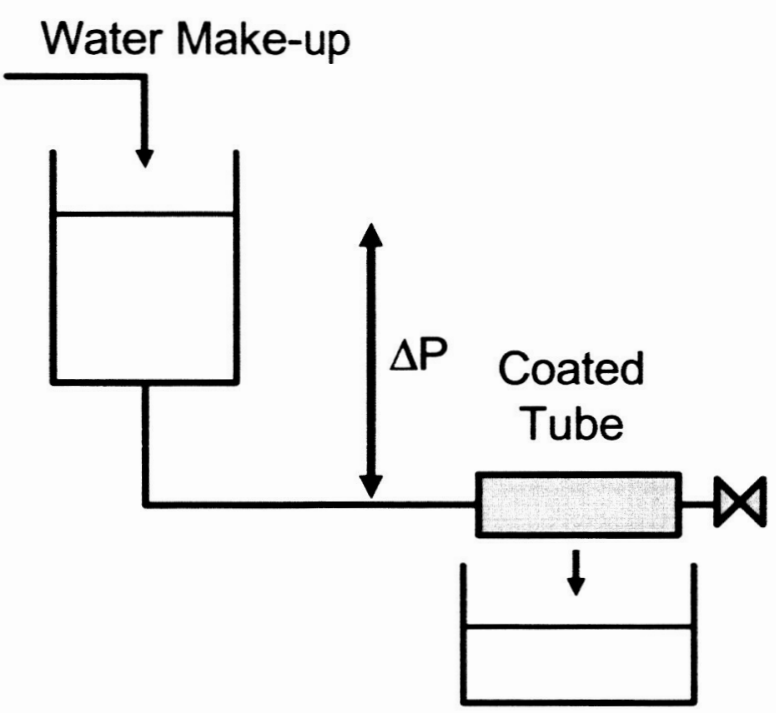

Figure 3: Water flux test set-up.

\section{Bubble Testing}

Ultimately, the coated membrane tubes will need to reject air bubbles while permitting permeation of water. Preliminary studies on bubble transport in wet systems were performed to determine the affect of flow rate for various coated membranes. The results were benchmarked against data for the uncoated tubes. Experiments were conducted by injection of air into the system as a slug. Retained air was collected in an inverted graduated cylinder.

\section{RESULTS AND DISCUSSION}

The existing gas traps used on the ISS employ two types of membranes. The hydrophilic membrane is composed of Nylon-11 (Figure 4a), and the hydrophobic membrane is composed of polypropylene. Relatively, the nylon is more hydrophilic; however, it is still a polymer, and inherently dislikes water. As a result, the pore size of 
these membranes $(3.6 \mu \mathrm{m})$ must be sufficiently large to accommodate the required water flux at a sufficiently low pressure drop. By changing to a more hydrophilic polymer, such as polyethersulfone (Figure 4b), a similar permeability can be obtained, but with much smaller pore size. Because homogeneous tubular membranes are not commercially available in the pore size needed for this application, we have devised a technique to make composite membranes by coating polyethersulfone on polyethylene. The wetted surfaces are polyethersulfone, and thus the desired permeation properties can be obtained, with the added stability of a rigid polyethylene support.

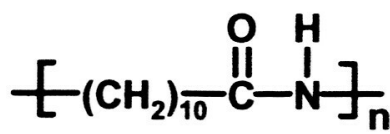

(a)

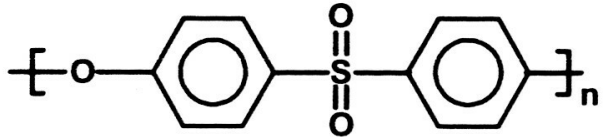

(b)

Figure 4. Chemical structures for (a) Nylon-11, and (b) Polyethersulfone (PES).

\section{Creation of Composite PES-PE Membranes}

Past experience with homogeneous PES membranes indicated that a moderately viscous polymer solution, properly degassed, can be used to create very uniform and repeatable membranes. In this study, differences in surface energies between the polymer film and substrate resulted in non-uniformities. Subsequent work has focused on improving interactions between the two materials, which has resulted in fairly repeatable and uniform coated tubes. The tubes do not yet have the desired properties; however, significant advancements have been made toward that end.

\section{Thick-Coated Membranes}

Initial studies were performed by coating dry tubes with a thick (10 g PES:45 mL DMF) polymer solution. Efforts were made to ensure even coating of the tube; however, pooling of the polymer solution generally resulted in significant inconsistencies in the coated membranes from these early studies. A comparison of the nitrogen flux behavior for the uncoated (upper line) and coated membranes is shown in Figure 5.

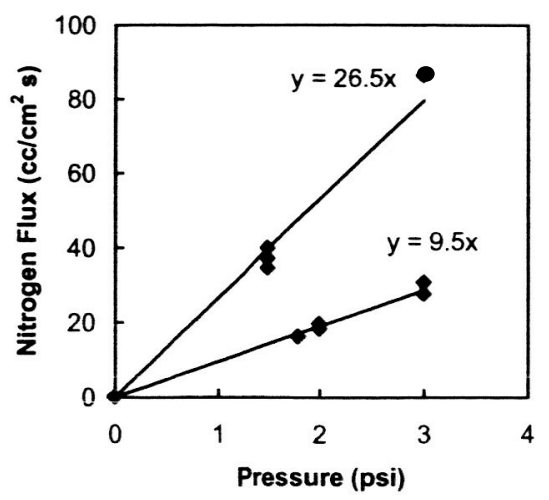

Figure 5: Nitrogen permeability decrease for the thick-coated composite membranes. 
The significant drop in membrane permeability is caused by a decrease in membrane pore size. This is shown more clearly in Figure 6. The bore surface of the raw PE tube is shown in Figure 6a, and the pore size of the PES layer is shown in Figure $6 \mathrm{~b}$. The PES creates a uniform layer with pores much smaller than the substrate, and provides the separating ability for bubble retention.
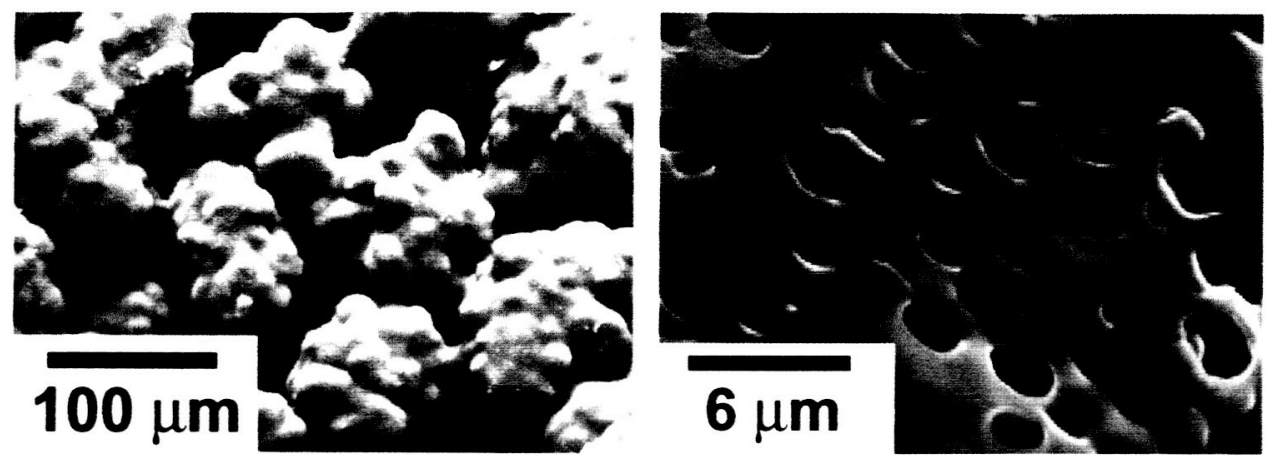

Figure 6: Raw PE tube (left) and surface of thick-coated membrane.

We were encouraged by these early results, but encountered problems with delamination of the added PES layer. Because the added layer was relatively thick, significant stresses were formed during drying, and the layer was pulled away from the rigid PE surface. A schematic of this phenomenon is shown in Figure 7. Thicker layers only enhanced this effect, and investigations were begun to examine how to improve interactions between the deposited layer and the support. Strategies have included increasing the surface energy of the support, reducing the viscosity of the PES coating solution, improving penetration of the PES layer, and increasing drain times to improve surface densification and to thin the added layer. The former was an effective technique to eliminate non-uniformities in the layer. Better interaction of the PES layer results in more fingering of the solution into the porous PE structure. This effect can be observed by examination of the back side of the added layer as in Figure 8 . Notice how where the surface was smooth (Figure 6), the back of the added layer is very rough from penetration into the PE structure. Thinning of the film was expected to preserve this effect, while reducing the stresses developed during drying.

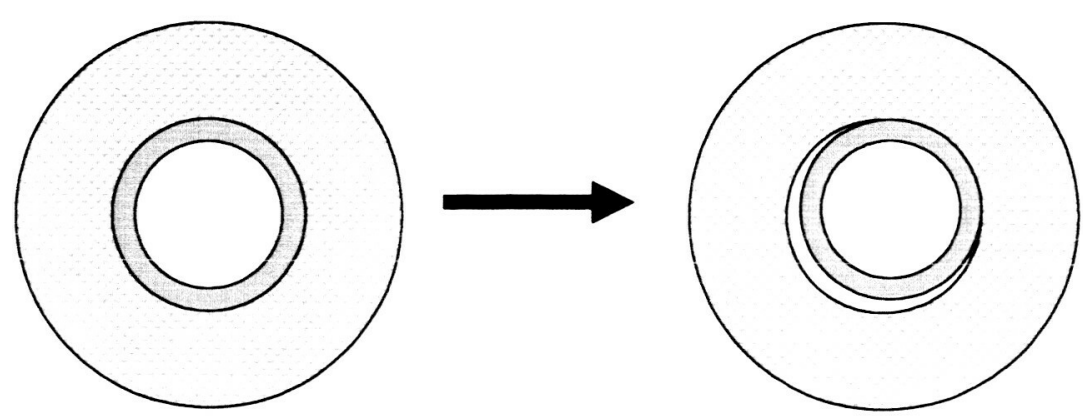

Figure 7: Delamination of thick added PES layer upon drying. 


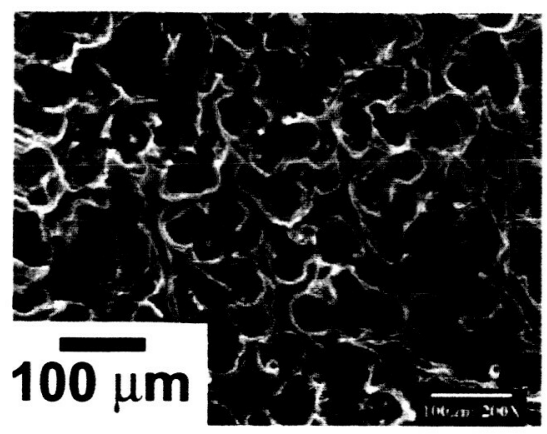

Figure 8: PES interface with PE tube after delamination.

\section{Thin-Coated Membranes}

Initial studies were performed to eliminate observed problems with the thick-coated membranes. Issues with delamination of the PES coating were eliminated by using a thinner PES solution (10g PES:90 mL DMF). Problems with pooling of excess polymer solution were eliminated by allowing the excess to drain and by wicking away residual solution with a paper towel. A representative SEM of the tube surface is shown in Figure 9.

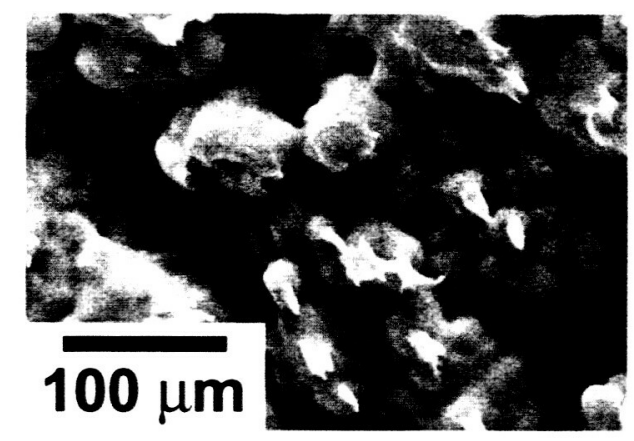

Figure 9: Thin-coated PES tube.

Notice the similarities to the raw membrane shown in Figure 6 . In this case, pore penetration is complete, and a distinct PES layer was not formed. There is evidence of PES seeding on the surface, which may be exploited during deposition of subsequent PES layers. The nitrogen flux behavior of the membranes was remarkably similar to the thick-coated membranes, indicating that although a distinct layer is not formed, the properties of the tube have been modified. A typical result for the thin-coated membrane is shown in Figure 10. In this case, the new permeability is approximately half of the raw tube permeability. The membrane permeability varied $\pm 30 \%$ for different thin-coated membranes. The most likely sources of error are the crosssectional area for nitrogen flow $( \pm 10 \%)$ and time keeping during testing $( \pm 10 \%)$. Secondary coating of PES generally resulted in an additional $33-75 \%$ decrease in nitrogen permeability and negligible problems with delamination. 


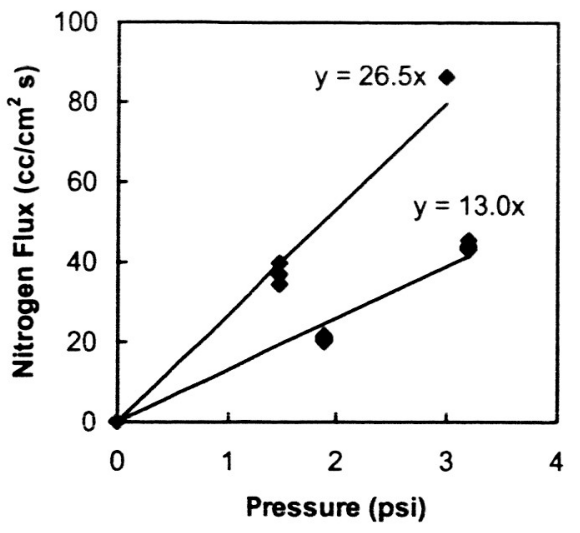

Figure 10: Representative nitrogen flux data for the thin-coated membranes (lower line) versus the uncoated tube.

\section{Thin-Soaked Membranes}

Intimate contact between the applied PES coated and the PE tube is critical to longterm stability of the composite material. In an effort to improve this contact, several membranes were soaked in the thin PES solution. The membranes had previously been soaked in DMF. It was hypothesized that long-term contact would allow more of the PES chains to diffuse into the PE tube porous structure. Representative nitrogen permeability data for the thin-soaked membrane is shown in Figure 11. The nitrogen permeability of these membranes was lower than all of the single-layer coated tubes. Repeatability was also improved, with $\pm 20 \%$ between all permeability values. There was no delamination of the applied PES.

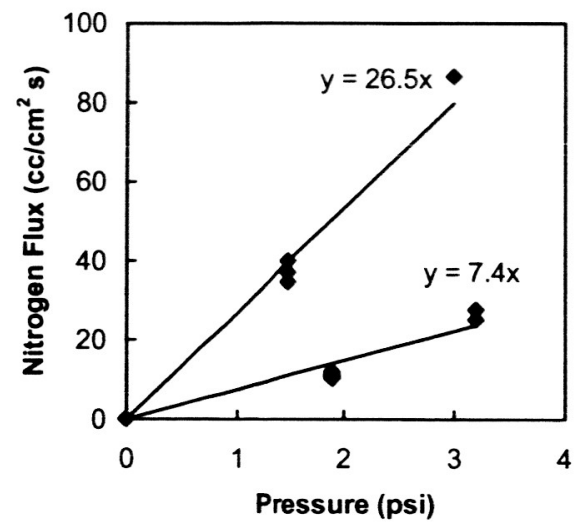

Figure 11: Typical nitrogen permeation data for the thin-soaked PES membranes (lower line) versus the uncoated tube.

\section{Water Contact and Swelling Effects}

Coated and soaked membranes were also permeated with water, followed by measurement of nitrogen permeability. In all cases, there was a significant decrease in nitrogen permeability. There may be two causes for this observed decrease. First, the 
PES coating may absorb water, causing it to swell. Much as inflammation will cause constriction of blood vessels, swelling of the polymer would constrict flow pathways, resulting in decreased nitrogen permeability. A second possibility is that water is retained in the porous structure. This is akin to the phenomenon that allows bubble retention in the gas trap. The nitrogen flux was measured multiple times for a wetted thin-soaked membrane. Over time, the nitrogen permeability improved and approached a steady value, as shown in Figure 12 . However, that permeability is still only $33 \%$ of the dry membrane permeability. Given that the water contact time is very small (about 1 minute) it is unlikely that a significant amount of water is absorbed. Rather, water in large pores is displaced at the permeation conditions ( 3.3 psid) and smaller pores retain water. The cross-sectional area for nitrogen flow is reduced, resulting in lower apparent permeability.

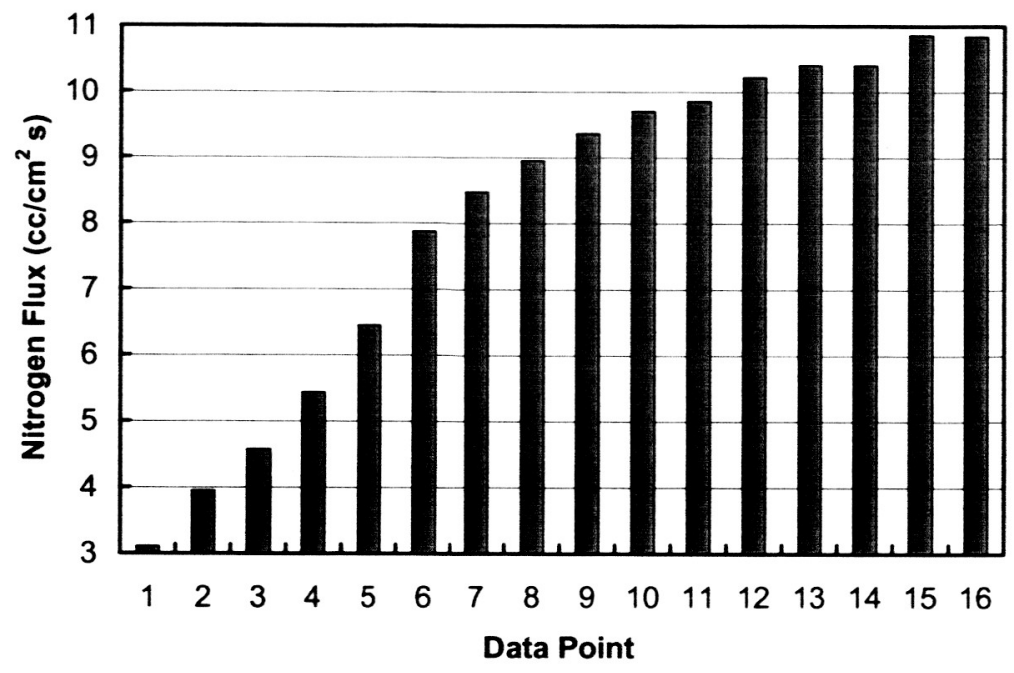

Figure 12: Partial nitrogen flux recovery for post-water contacted thin-soaked membrane.

\section{Membrane Thinning through Increased Drain Times}

Although the thin-coated and thin-soaked membranes gave favorable permeation properties with no delamination, they may encounter difficulties due to surface roughness (recall Figure 9). The potential for gas bubbles to be trapped and subsequently permeating the membrane must be considered, and thus the formation of a distinct layer, such as in Figure 6, would be desirable. The formation of a thin layer eliminated delamination problems, but did not result in a distinct layer. Therefore the coating procedure was modified to create a thin coating using the thicker solution by increasing the polymer drain time prior to phase inversion. An SEM micrograph showing the surface of a tube coated in this manner is shown in Figure 13. The extended drain time not only results in a thinner coating and eliminates delamination, but the pore size of the membrane is also smaller. In this case, the pore size is approximately $0.14 \mu \mathrm{m}$. The improved coated tubes also exhibited excellent water permeation behavior, with an average permeability of $167-206 \mathrm{lb} / \mathrm{ft}^{2} \mathrm{hr}$ psi. The current membrane gas traps using Nylon-11 membranes with a pore size of $3.6 \mu \mathrm{m}$ have nearly the same permeability. This exciting result shows great promise for replacement of the 
existing technology with PES coated tubes that are reproducible, made with commercially available materials, and operate within existing pressure drop, flow rate, and footprint specifications.

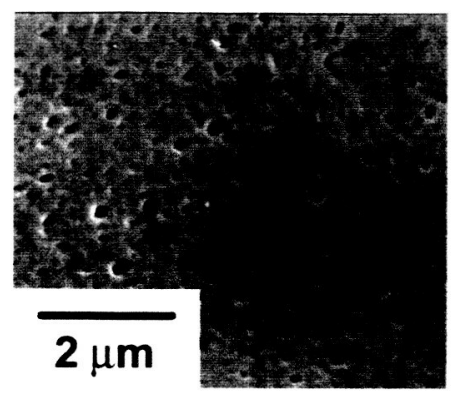

Figure 13: SEM micrograph of thick-coated PES membrane with extended drain time.

\section{Preliminary Bubble Retention Studies}

Adequate coating stability and water flux properties must be matched by good retention of gas bubbles. Preliminary studies have been performed in this area by injection of 50 $\mathrm{cm}^{3}$ of air into the system shown in Figure 14. Water is flowing prior to air injection, so the pores of the coated tubes are filled with water. Air must displace this water or it will be collected in the graduated cylinder. All studies were performed at a pressure drop of $1.72 \mathrm{psi}$. The tube was oriented parallel to the bench top, so that injected air would have good accessibility to the membrane surface. The results showed that for low liquid flow rates, $100 \%$ of the injected air permeated the walls of the uncoated tube. Some air was retained at higher flow rates. In the case of the PES coated tube, $100 \%$ of the injected air was retained at all flow rates. Although we have not shown venting of the retained gas bubbles through the hydrophobic membrane, these results do offer preliminary evidence that gas bubbles will be retained by the PES coated tubes.

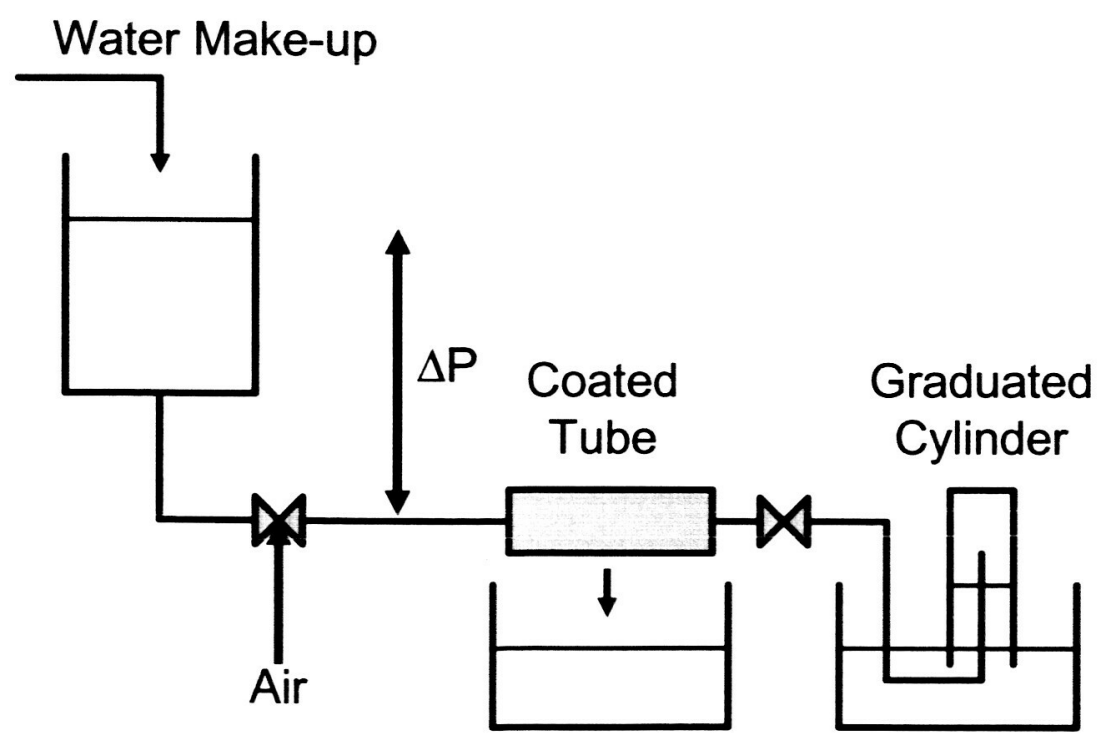

Figure 14: Bubble test set-up. 


\section{CONCLUSIONS AND FUTURE DIRECTIONS}

Deposition of a layer of PES on the bore surface and in the porous structure of a PE tube will alter the tube permeability. Optimization of the coating process is imperative to create reproducible membranes with desired characteristics. Thick polymer coatings are ineffective because of inconsistencies and material disconnects between the dried polymer coating and the substrate. These issues have been addressed by presoaking the PE tube in the solvent to increase the surface energy at the interface. The use of a thinner solution was found to generate membranes with similar permeability characteristics, but without delamination. However, their surface was very rough (similar to raw tube) and may create opportunities for gas leakage. A combination of tube presoaking and extended draining time resulted in coated tubes with nearly the same permeability as Nylon-11 membranes used in the current gas trap design. The new coated tubes, however, have a smaller pore size, and should provide a higher bubble pressure. Preliminary studies on bubble retention showed a dramatic improvement over the uncoated tube, with $100 \%$ of air bubbles retained at a pressure drop of $1.72 \mathrm{psi}$. Future work will address integration and reconfiguration of the hydrophobic membrane for gas venting.

\section{ACKNOWLEDGEMENTS}

The authors recognize NASA and the NASA Summer Faculty Fellowship Program for financial support of this work. Thanks are also due to the Pressurized Carriers Group/FD24 at MSFC. Lab space was provided by FD21/MSFC, with special help from Gene Hartsfield and Bill Barnett. SEM pictures were provided with the help of James Coston in ED33/MSFC, and Tapan Shah at the University of Alabama.

\section{REFERENCES}

Faust, M.B.; Zhou, S.J.; Brubaker, I.M., "Dual-membrane gas trap for the Space Station Freedom internal thermal control system," SAE Technical Paper Series 932162, presented at the $23^{\text {rd }}$ International Conference on Environmental Systems, Colorado Springs, CO, July 1993.

Ritchie, S.M.C.; Holladay, J.B.; Holt, J.M.; Clark, D.W., "An improved design for air removal from aerospace fluid loop coolant systems," SAE Technical Paper Series 2003-01-2569, presented at the $33^{\text {rd }}$ International Conference on Environmental Systems, Vancouver, BC, July 2003. 